\title{
Femtosecond-Laser-Induced Defects on Silicon Carbide Probed by Electrical Conductivity
}

\author{
Takuro Tomita*, Manato Deki*,***, Eizo Yanagita*, Yota Bando*, Yoshiki Naoi*, \\ Takahiro Makino** and Takeshi Ohshima** $^{* *}$ \\ *Faculty of Engineering, Tokushima University, \\ 2-1 Minamijosanjima, Tokushima, Japan \\ E-mail: tomita@tokusima-u.ac.jp \\ ** National Institutes for Quantum and Radiological Science and Technology, \\ Watanukimachi, Gunma, Japan \\ **** Present address: Center for Integrated Research of Future Electronics (CIRFE), \\ Institute of Materials Systems for Sustainability (IMaSS), Nagoya University, \\ Chikusa-ku, Nagoya Japan
}

\begin{abstract}
The temperature dependence of the electrical conductivity of a femtosecond-laser-modified region in silicon carbide has been examined. The current-voltage characteristics of the modified region showed ohmic characteristics. The specific resistivity exhibited a dramatic decrease of four orders of magnitude in response to a temperature increase of 80 to $400 \mathrm{~K}$. The temperature dependence of the specific resistance was successfully fit by a double exponential curve, indicating two defect levels. This result means that two types of levels were induced by the laser irradiation. The irradiation fluence dependence of the energies of the two levels and the population ratios between them are discussed herein.
\end{abstract}

DOI: 10.2961/jlmn.2017.02.0004

Keywords: femtosecond laser, silicon carbide, electrical conductivity, temperature dependence, modification

\section{Introduction}

The potential applications of femtosecond laser irradiation are attracting considerable attention from a wide variety of industrial fields. For example, a femtosecond laser can be used to cut metals, semiconductors, and glass with a high spatial precision of a few hundreds of nanometers. Recently, femtosecond laser irradiation has also seen initial applications in the biomedical field; for example, in laser in-situ keratomileusis (LASIK) [1]. With a view to understanding the fundamental mechanism of femtosecond laser processing, several studies have been conducted [2]. For example, Tinen et al. have reported the results of ablation dynamics investigations on semiconductors and metals performed using the Linnik interferometer [3]. According to their observations, the exfoliated films expand and form a dome which continues to expand to approximately $1 \mathrm{~ns}$. We have also observed the initial process of femtosecond laser ablation by applying a soft x-ray laser probe to several irradiated metals $[4,5]$. The results show that the thin film is exfoliated from the surface of the substrate after the laser irradiation. Then, the exfoliated film expands toward the normal relative to the sample surface, i.e. in the opposite direction to the incident laser beam. Interestingly, the expanded shape of the exfoliated films corresponds to the lateral intensity profile of the incident laser beam [6]. In addition, the vacancies in the silicon carbide (SiC) substrate are attracting considerable interest, because this ma- terial can be subjected to a single photon source [7-9], which can be applicable to quantum spintronics and photonics. To realize quantum technology based on SiC substrate, the development of a femtosecond modification technique, especially one facilitating local control of the electrical conductivity is quite important. This aspect is partially noteworthy because the transport of spin carriers is one of the most fundamental issues influencing the development of quantum devices. Previously, we have reported on the electrical conductivity observed for femtosecondlaser-modified SiC [10, 11]. It was found that the electrical conductivity exhibits a dramatic increase above the threshold fluence [10] following laser modification, which indicates that the femtosecond laser modification technique can be applied to control the local electrical conductivity of SiC. However, the origin of the higher conductivity has not been discussed. It has also been found that the electrical conductivity in the laser-modified region exhibits temperature dependence, indicating the existence of trapping levels $[11,12]$. If an understanding of the nature of these trapping levels is obtained, it is highly possible that the femtosecond laser modification technique will become realized as a tool for drawing electrical circuits both inside and on the surface of SiC. However, some issues require clarification. To realize quantum devices using the femtosecond laser modification technique, one of the most important issues is the maximum operation temperature of the electrical circuits fabricated using the femtosecond laser irradiation. There- 
fore, in this study, we discuss the electrical conductivity in a modified region of an $\mathrm{SiC}$ specimen for a wide range of ambient temperatures in order to reveal the energy levels and carrier population. This insight provides essential information on the carrier transport properties.

\section{Experimental}

We used semi-insulating $6 \mathrm{H}-\mathrm{SiC}$ single crystal substrates (SiXON, Japan) with a specific resistance of $\rho>10^{5}$ $\Omega \mathrm{cm}$. We conducted three-fold phosphorus (P) ion implantation to fabricate ohmic contact on the semi-insulating SiC. The averaged phosphorous density beyond the depth of approximately $150 \mathrm{~nm}$ beneath the surface was approximately $5 \times 10^{19} \mathrm{~cm}^{-3}$. For the metal contacts, aluminum (Al) was evaporated on the ion-implanted area in the vacuum chamber. The distance between the ion-implanted metal contacts was approximately $1.2 \mathrm{~mm}$. We used a Ti:sapphire regenerative amplifier (Spitfire, Spectra Physics, USA) for the light source. The wavelength was $800 \mathrm{~nm}$ and the pulse duration, which was evaluated by a single-shot autocorrelator, was $130 \mathrm{fs}$. The pulse laser was operated at a repetition rate of $1 \mathrm{kHz}$. To observe the irradiation on the SiC surface, we used an inverted optical microscope (IX-70, Olympus, Japan) and a 10X objective lens with a numerical aperture of approximately 0.40 . The radius of the beam focused on the sample surface was $2.1 \mu \mathrm{m}$.

During the laser irradiation, the sample was constantly translated at a rate of $100 \mu \mathrm{m} / \mathrm{s}$ by using a mechanical motorized stage (KS701-20LHD, Suruga Seiki, Japan). The irradiation was performed between the $\mathrm{Al}$ contacts, and the electric field of the incident laser beam was parallel to the scanning direction. The laser fluences were varied from 2.9 to $9.4 \mathrm{~J} / \mathrm{cm}^{2}$ and the current-voltage (I-V) characteristics on the laser-modified lines were measured. The applied voltage was from -100 to $+100 \mathrm{mV}$ with $10-\mathrm{mV}$ resolution. The temperature of the sample was reduced to $77 \mathrm{~K}$ using liquid nitrogen, and the sample temperature was controlled

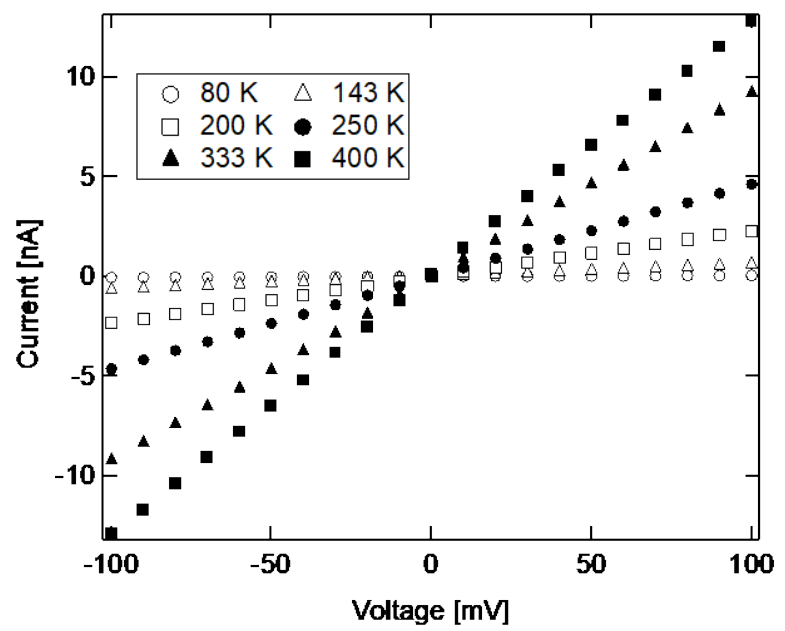

Fig. 1 Current-voltage (I-V) characteristics of femtosecond-modified region with irradiation fluence of $9.4 \mathrm{~J} / \mathrm{cm}^{2}$. The temperature was varied from 80 to 400 K. Current-voltage (I-V) characteristics of femtosecond-modified region with irradiation fluence of $9.4 \mathrm{~J} / \mathrm{cm} 2$. The temperature was varied from 80 to $400 \mathrm{~K}$. from 80 to $400 \mathrm{~K}$.

\section{Results and Discussion}

The $I-V$ characteristics of the laser-modified areas in the $\mathrm{SiC}$ are shown in Fig. 1. The irradiated fluence was 9.4 $\mathrm{J} / \mathrm{cm}^{2}$, which is above the threshold fluence for the dramatic decrease in the resistance [10]. The I-V characteristics exhibited ohmic characteristics for all the examined temperature, i.e. 80 to $400 \mathrm{~K}$. It was clearly found that the slope increased significantly with increasing temperature. Although the result is not shown, the I-V characteristics for the sample prior to laser irradiation were consistently less than 1 pA up to an applied voltage of $+100 \mathrm{mV}$, yielding a trendline that was almost colinear to the abscissa. This tendency was almost identical for all the examined temperatures. Therefore, it is obvious that the observed finite conductivity and the temperature dependence were induced by the femtosecond laser modification.

The temperature dependence of the electrical conductivity for various fluence values is shown in Fig. 2. In the electrical conductivity estimation, a space with dimensions of 4.2- $\mu \mathrm{m}$ width and $50-\mathrm{nm}$ depth was used as the modified region; these dimensions were obtained from the optical microscope observation and transmission electron microscopy (TEM) measurement, respectively [13]. Although the femtosecond laser irradiated spot is ablated with the depth of submicron scale [13], the modification of the irradiated material occurs at the bottom of the crater [14]. Thus, it is supposed that the modification at the bottom of the crater is the origin of the electrical conductivity. The electrical conductivity monotonically increased as the temperature increased from 80 to $400 \mathrm{~K}$. This means that the increase in the electrical conductivity was induced by the carriers, which were excited by phonons. This results indicate that the laser-modified SiC has a semiconducting property. In addition, the electrical conductivity increased with increasing irradiation fluence.

To discuss the physics of the carrier conduction, we estimated the activation energies of each level in the modified SiC. In order to analyze these data, we employed the Arrhenius conduction model. The data was fitted according

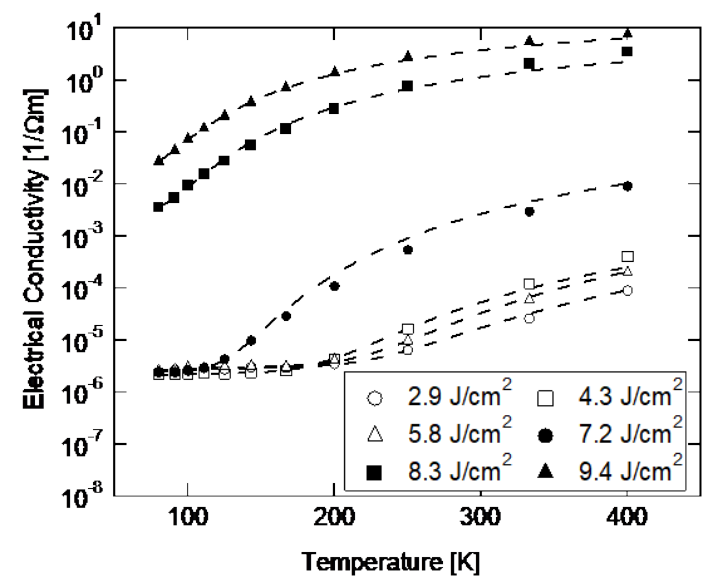

Fig. 2 Temperature dependence of specific resistivity of femtosecond-laser-modified region for various irradiation conditions. The fitting results obtained by assuming two defect levels are indicated by broken lines. 
to

$$
\sigma=\sigma_{1} e^{-\frac{E_{D 1}}{k_{B} T}}+\sigma_{2} e^{-\frac{E_{D 2}}{k_{B} T}}
$$

Here, $\sigma$ is the electrical conductivity, $E_{D 1}$ and $E_{D 2}$ are the activation energies of the defect levels, respectively, $\sigma_{1}$ and $\sigma_{2}$ are the electrical conductivities of each of these levels, respectively, $k_{B}$ is Boltzmann's constant, and $T$ is the sample temperature. It should be noted that the values of $\sigma_{1}$ and $\sigma_{2}$ are directly related to the impurity population. In the fitting procedure, the values of $E_{D 1}, E_{D 2}, \sigma_{1}$, and $\sigma_{2}$ were used as the fitting constants. In Fig. 2, the fit result for each irradiation fluence is indicated by a broken line. For all the examined temperatures, the experimental results were well fit by assuming that the constants $\sigma_{1}$ and $\sigma_{2}$ were non-zero components, indicating that the two defect levels exist in the laser-modified areas. In general, electrical conduction in amorphous semiconductors can be classified into three mechanisms: the conduction in the extended states, in the band tail state, and in the localized states at the Fermi level [15]. As regards the Arrhenius conduction, the carriers are thermally excited in the extended state. Therefore it is also indicated that the temperature dependence of the femtosecond-laser-modified region exhibited Arrhenius behavior [16].

From the fitting results, the values of $E_{D 1}$ and $E_{D 2}$ were successfully obtained for each irradiation fluence. These values are plotted in Fig. 3 using open symbols (left ordinate). The values of $\sigma_{1}$ and $\sigma_{2}$ are also plotted in this figure using by closed symbols (right ordinate). The values of $E_{1}$ and $E_{2}$ are almost constant at approximately 2 and $200 \mathrm{meV}$, respectively, below $8 \mathrm{~J} / \mathrm{cm}^{2}$. Above $8 \mathrm{~J} / \mathrm{cm}^{2}$, the values of $\mathrm{E}_{1}$ and $\mathrm{E}_{2}$ increase and decreases, respectively. Simultaneously, the values of $\sigma_{1}$ and $\sigma_{2}$ increase for fluence of more than $8 \mathrm{~J} / \mathrm{cm}^{2}$. The growth in the values of $\sigma_{1}$ and $\sigma_{2}$ indicates that the number of thermally excited carriers is increased, i.e. that the number of defect levels is increased.

Previously we reported the results of Raman spectroscopy and TEM observation for femtosecond-laser-modified $\mathrm{SiC}$, indicating the formation of a phase consisting of amorphous silicon and amorphous carbon after laser irradiation. In addition, we observed an amorphous silicon carbide phase obtained under higher irradiation conditions

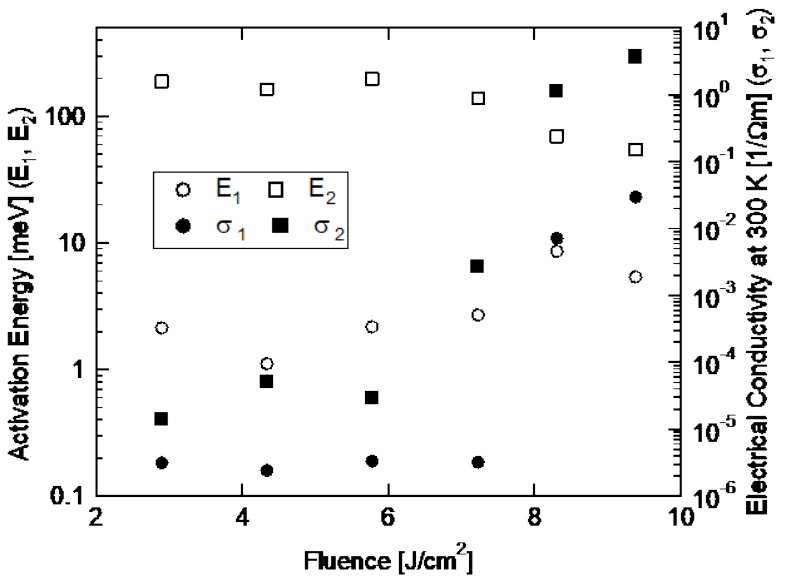

Fig. 3 Temperature dependence of activation energy and specific resistivity of each defect level in femtosecond-laser-modified region.
[14]. The threshold fluence for the formation of the amorphous silicon carbide was approximately $0.5 \mathrm{~J} / \mathrm{cm}^{2}$, and that for the amorphous silicon and carbon was approximately $1.3 \mathrm{~J} / \mathrm{cm}^{2}$. Although these values depend on the irradiation conditions, their order is roughly constant. Thus, the fluence for which the significant electrical conductivity occurs is far above the threshold fluence for the formation of amorphous silicon carbide, silicon, and carbon. Therefore, the formation of the amorphous phase material is not directly related to the change in the electrical conductivity. Instead, we assume that piling and coalescence of these materials constitute the origin of the electrical conductivity. If we assume that the average distance between each defect site is $10 \mathrm{~nm}$, the corresponding defect density is $\mathrm{n}=10^{18}$ $\left(1 / \mathrm{cm}^{3}\right)$. On the other hand, $\mathrm{n}=10^{15}\left(1 / \mathrm{cm}^{3}\right)$ for a distance of $100 \mathrm{~nm}$. It is supposed that significant interaction or coalescence between each site emerges for average distances ranging from 10 to $100 \mathrm{~nm}$. It is also probable that the electric conductivity significantly increases for carrier densities ranging from $10^{18}$ to $10^{15}\left(1 / \mathrm{cm}^{3}\right)$. This explains why the activation energies of $E_{1}$ and $E_{2}$ change for higher fluence.

\section{Conclusion}

The temperature dependence of a femtosecond-lasermodified region on $\mathrm{SiC}$ was measured in this study. The $I-V$ characteristics showed ohmic properties. The temperature dependence of the specific resistance was well fit by double exponential curves. The irradiation fluence dependence of the trapping energy was also obtained. These defect levels were related to two phases, both of which form following femtosecond laser irradiation. One phase consists of amorphous silicon carbide, whereas the other consists of amorphous silicon and amorphous carbon. The effect of the interactions between each site was also observed for higher irradiation conditions.

\section{Acknowledgments}

This work was partially supported by a JSPS Grant-inAid for Challenging Exploratory Research (26630132), a Grant-in-Aid for Scientific Research (B) (25286086), and a Grant-in-Aid for Scientific Research on Innovative Areas (24110712). This work was also partially supported by the Nippon Sheet Glass Foundation (NSG Foundation) for Materials Science and Engineering and the AMADA foundation.

\section{References}

[1] G. M Kezirian and K. G Stonecipher: J. of Cataract \& Refractive Surgery, 30, (2004) 804.

[2] V. V. Temnov, K. Sokolowski-Tinten, P. Zhou, and D. Linde: J. Opt. Soc. Am. B, 23, (2006) 1954.

[3] K. Sokolowski-Tinten, J. Bialkowski, A. Cavalleri, D. Linde, A. Oparin, J. M. Vehn, and S. I. Anisimov: Phys. Rev. Lett., 81, (1998), 224.

[4] T. Tomita, M. Yamamoto, N. Hasegawa, K. Terakawa, Y. Minami, M. Nishikino, M. Ishino, T. Kaihori, Y. Ochi, T. Kawachi, M. Yamagiwa, and T. Suemoto: Opt. Express, 20, (2012) 29329.

[5] T. Tomita, M. Nishikino, N. Hasegawa, Y. Minami, R. Takei, M. Baba, T. Eyama, S. Takayoshi, T. Kaihori, T. Morita, Y. Hirano, T. Kawachi, M. Yamagiwa, and T. 
Suemoto: J. of Laser Micro Nanoeng., 9, (2014) 137.

[6] N. Kakimoto, T. Eyama, R. Izutsu, and T. Tomita: J. of Laser Micro Nanoeng., 11, (2016) 91.

[7] S. Castelletto, B. C. Johnson, V. Iv'ady, N. Stavrias, T. Umeda, A. Gali, and T. Ohshima: Nat. Materials, 13, (2014) 151.

[8] D. J. Christle, A. L. Falk, P. Andrich, P. V. Klimov, J. U. Hassan, N. T. Son, E. Janz'en, T. Ohshima, and D. D. Awschalom: Nat. Materials, 14, (2015) 160.

[9] M. Widmann, S.-Y. Lee, T. Rendler, N. T. Son, H. Fedder, S. Paik, L.-P. Yang, N. Zhao, S. Yang, I. Booker, A. Denisenko, M. Jamali, S. A. Momenzadeh, I. Gerhardt, T. Ohshima, A. Gali, E. Janzen: Nat. Materials, 14, (2015) 164.

[10] M. Deki, T. Ito, M. Yamamoto, T. Tomita, S. Matsuo, S. Hashimoto, T. Kitada, T. Isu, S. Onoda, and T. Ohshima: Appl. Phys. Lett., 98, (2011) 133104.

[11] M. Deki, T. Oka, S. Takayoshi, Y. Naoi, T. Makino, T. Ohshima, and T. Tomita: Mat. Sci. Forum, 778, (2014) 661.

[12] T. Tomita, M. Iwami, M. Yamamoto, M. Deki, S. Matsuo, S. Hashimoto, Y. Nakagawa, T. Kitada, T. Isu, S. Saito, K. Sakai, S. Onoda, and T. Ohshima: Mat. Sci. Forum, 645, (2010) 239.

[13] T. Okada, H. Kawahara, Y. Ishida, R. Kumai, T. Tomita, S. Matsuo, S. Hashimoto, M. Kawamoto, Y. Makita, and M. Yamaguchi: Appl. Phys. A, 92, (2008) 665.

[14] M. Yamaguchi, S. Ueno, R. Kumai, K. Kinoshita, T. Murai, T. Tomita, S. Matsuo, and S. Hashimoto: Appl. Phys. A, Materials Science \& Processing, 99, (2010) 23.

[15] X. Shi, H. Fu, J. Shi, L. Cheah, B. Tay, and P. Hui: J. of Phys.: Condensed Matter, 10, (1998) 9293.

[16] G. Lazar, K. Zellama, M. Clin, and C. Godet: Appl. Phys. Lett., 85, (2004) 6176.

(Received: April 3, 2017, Accepted: June 3, 2017) 